\title{
Hollow Core Fibres for High Capacity Data Transmission
}

\author{
F. Poletti*, M.N. Petrovich*, N.V. Wheeler*, N.K. Baddela*, E. Numkam Fokoua*, J.P. \\ Wooler*, D.R. Gray*, S.R. Sandoghchi*, J.R. Hayes*, Y. Jung*, R. Slavík*, S.U. Alam*, \\ V.A.J.M. Sleiffer**, M. Kuschnerov***, and D.J. Richardson* \\ * Optoelectronics Research Centre, University of Southampton, Highfield, Southampton, SO17 1BJ, UK \\ ** COBRA institute, Eindhoven University of technology, Eindhoven, The Nederlands \\ *** Coriant R\&D GmbH, St-Martin-Str. 76, Munich, Germany
}

\begin{abstract}
We review our progress in developing, characterizing and handling hollow-core photonic bandgap fibers with improved transmission properties, targeted at high-capacity, low-latency data transmission in the current telecoms window and at the potentially lower-loss $2 \mu \mathrm{m}$ wavelengths.
\end{abstract}

\section{INTRODUCTION}

One of the most radical approaches to address the fundamental limits of loss and nonlinearity in transmission fibers is arguably represented by the use of hollow core Photonic Bandgap Fibers (PBGFs) [1]. The periodic photonic crystal cladding in these structures provides tight, wavelength-specific light guidance in an air-filled hollow core. As the overlap with the glass structure is only fractional, these fibres readily offer orders-of-magnitude reduction of the optical nonlinearity, ultimate low-latency (close to the vacuum speed of light) and potential for ultra-low transmission loss.

In this work we will present our progress in developing PBGFs suitable for high capacity data transmission.

\section{WIDE BANDWIDTH OPERATION THROUGH SURFACE MODE ELIMINATION}

One of the first problems that needs to be addressed in the development of PBGFs for high capacity telecoms applications is that of producing low loss fibres which do not support surface modes (SMs). SMs, physically located at an incorrectly terminated or distorted core surround, typically interact with the various air-guided core modes at different spectral positions within the low loss bandgap region. This significantly reduces the useable transmission bandwidth and makes the whole fibre extremely sensitive to external perturbations [2].

We have recently demonstrated a fabrication recipe for low loss fibres based on the use of a large ( 19 cell) core
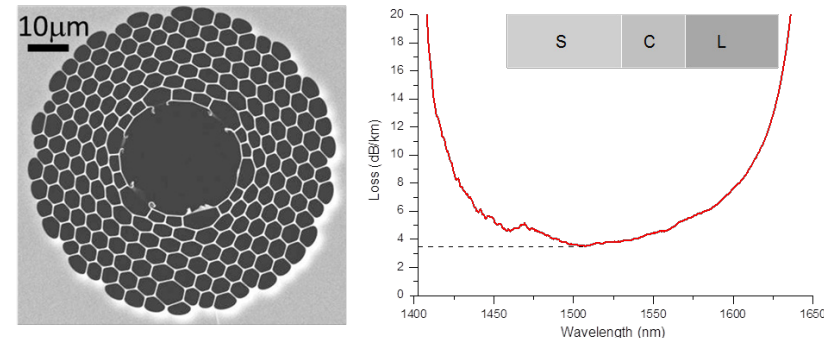

Fig. 1. SEM (right) and cutback loss (left) of a 19 cell PBGF with a minimum loss of $3.5 \mathrm{~dB} / \mathrm{km}$ and a $3 \mathrm{~dB}$ bandwidth of $160 \mathrm{~nm}$, [4] with a thin core surround [3] and a carefully controlled core boundary, which allows us to reproducibly fabricate fibres with a SM-free bandwidth in excess of $150 \mathrm{~nm}$ and losses well below $5 \mathrm{~dB} / \mathrm{km}$ [4], see Fig. 1 .

Investigation of the modal content of fibres fabricated with this method through both $\mathrm{S}^{2}$ and time of flight techniques indicates the presence of distinct high order modes. All modes are free from anticrossings with SMs throughout the whole C-band, and the level of intermodal cross-talk over the few hundred meters tested is below the experimentally measurable level, Fig.2a,b.
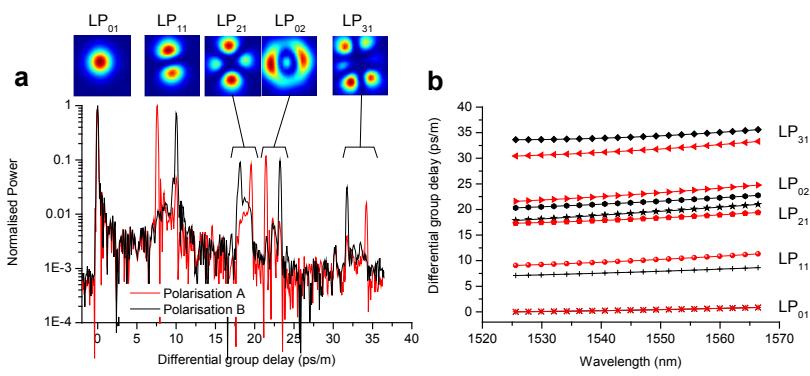

C
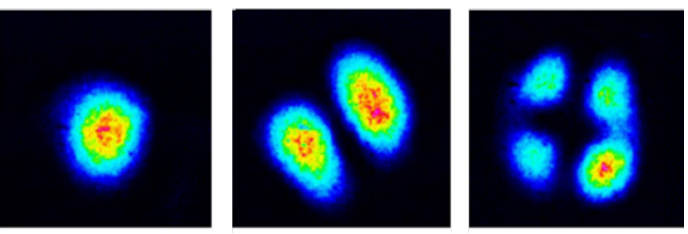

Fig. 2. (a) Time of flight measurement for the fibre in Fig.1 indicating 5 well-separated mode groups; (b) Wavelength dependence of the DGD of all modes showing no evidence of SM interactions; (c) Example of individual modes transmitted through $230 \mathrm{~m}$ of fibre.

\section{DATA TRANSMISSION EXPERIMENTS}

Using mode multiplexing techniques it is possible to excite and transmit several modes through the fibre (see Fig. 2c), which opens up the potential for further capacity expansion through space division multiplexing (SDM) schemes. On the other hand, by virtue of the low intermodal cross-talk, these fibres can also be operated as effectively single mode. Through selective excitation of the fundamental mode and spatial filtering at the receiver end we have recently demonstrated transmission of $37 \times 40$ Gbit/s DWDM on-off keyed channels in the C-band over $250 \mathrm{~m}$ of the fibre shown in Fig.1 [5].

Encouraged by the low power penalties in this experiment we have then further increased the overall transmitted capacity by exploiting both polarisations, 
increasing the number of DWDM channels and using more spectrally efficient coherent modulation formats. In this way we have demonstrated up to $30.7 \mathrm{Tbit} / \mathrm{s}$ using 32QAM (2x96x160 Gbit/s/channel/polarisation) which, to the best of our knowledge, is currently the highest data rate ever transmitted through a PBGF [6].

It is well known that the $\lambda^{-3}$ scattering loss dependence and the lower infrared glass absorption of PBGFs push their minimum predicted loss region to wavelengths around $2 \mu \mathrm{m}$ [7]. In order to investigate the potential of data transmission at these novel wavelengths and address related issues we have fabricated PBGFs operating in this spectral region. Fig. 3 shows the cutback loss of a fibre with a minimum loss of $4.5 \mathrm{~dB} / \mathrm{km}$ at $1980 \mathrm{~nm}$ and a bandwidth well matched to that of a custom built Thulium doped amplifier (TDFA). Using commercially available transmission components we have recently reported the first data transmission through a PBGF at these wavelengths [8]. While clearly not comparable to the impressive capacity achievable in the $\mathrm{C}$-band due to the relatively much less mature component technology, these early experiments demonstrate that should the PBGFs loss be reduced by a further factor of $\sim 10$ or so, which seems realistic, no major technological road blocks currently appear to exist which will ultimately prevent data transmission in the $2 \mu \mathrm{m}$ wavelength region.

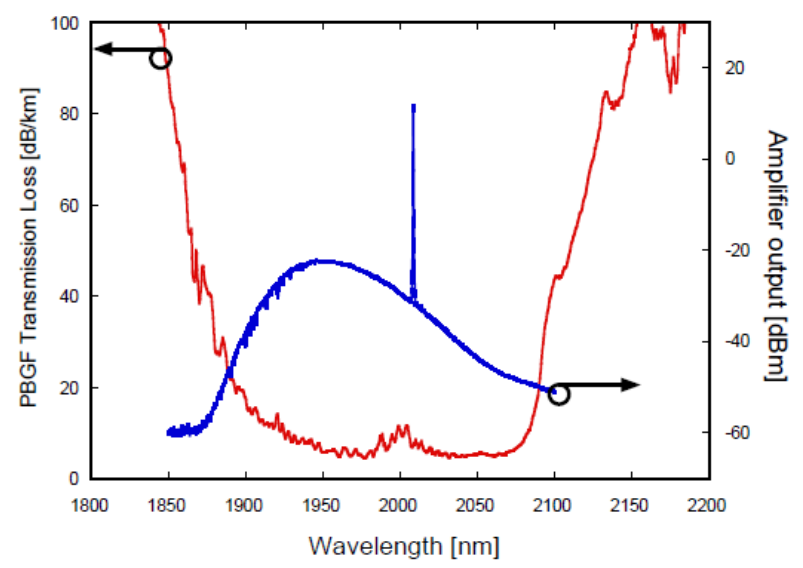

Fig. 3. Cutback loss of a $2 \mu \mathrm{m}$ operating PBGF with a minimum loss of $4.5 \mathrm{~dB} / \mathrm{km}$ (red curve) and a $150 \mathrm{~nm}$ bandwidth matched to the ASE emission of a custom built TDFA (blue curve).

\section{PbGf-Pbgf Splicing}

Apart from a further reduction in the loss, another mandatory requirement for PBGFs to be practically useable as transmission fibres is the possibility to be selfspliced with low loss. Although in principle this is a challenging problem to solve as it requires applying sufficient heat to fuse the solid cladding together without causing collapse in the thin microstructured cladding, we have recently demonstrated a way to achieve this. Using a sequence of tack, sweep and short arc pulses with a standard fusion splicer we have achieved robust splices with an average loss of $0.16 \mathrm{~dB}$ with significant potential for further improvement (Fig.4) and a negligible impact on the fibre's high order mode content. No significant power penalty was observed in data transmission over PBGFs containing splices fabricated with this recipe [9].
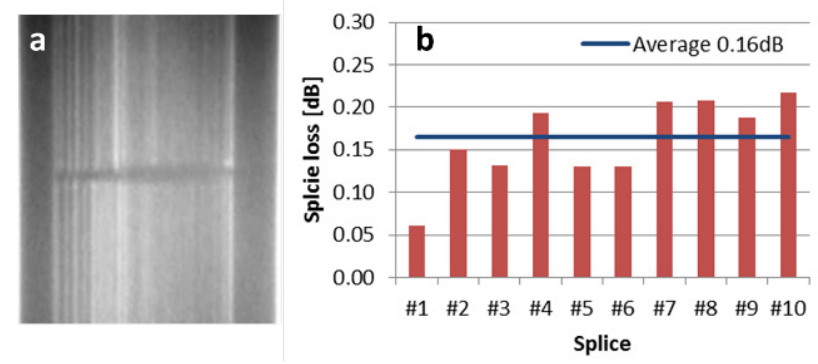

Fig. 4. (a) Two PBGFs spliced with the multi-step recipe of Ref. [9] showing no distortion in the microstructure and $\mathrm{a} \sim 10 \mu \mathrm{m}$ residual air gap; (b) Splice loss for 10 different realizations (min: $0.07 \mathrm{~dB}$, average $0.16 \mathrm{~dB})$.

\section{Conclusions}

We have presented some of our latest results in the development of hollow core PBGFs suitable for high capacity data transmission. Although much work remains to be done for this technology to become of practical interest for data transmission, over the last two years we have demonstrated significant progress in this area. Fibres with an uninterrupted transmission bandwidth covering the $\mathrm{S}+\mathrm{C}+\mathrm{L}$ bands can be realized; once surface modes are eliminated, the air-guided modes of these PBGFs are "well behaved" and with sufficiently low intermodal cross-talk to allow for single mode and potentially even SDM transmission; all components enabling data transmission in the lowest loss $2 \mu \mathrm{m}$ wavelength region are already available and all performance metrics are expected to rapidly improve with further development in the ever expanding $2 \mu \mathrm{m}$ component market; and finally, PBGFs can be robustly interconnected to themselves with low loss using a conventional arc-fusion splicer and a multi-step splicing procedure.

\section{ACKNOWLEDGMENT}

This work was supported by the EU 7th Framework Programme under grant agreement 258033 (MODE-GAP) and by the UK EPSRC through grant EP/I01196X/1 (HYPERHIGHWAY).

\section{REFERENCES}

[1] R. F. Cregan et al., Science, 285, 1537-1539, (1999)

[2] F. Poletti, et al., Optics Express, 13, 9115-9124, (2005)

[3] R. Amezcua-Correa, et al., Optics Express 15, 17577 17586 (2007)

[4] N. V. Wheeler et al., Proc OFC'12, PDP5A.2.

[5] R. Slavik, et al., Proc ECOC'12, Mo.2.F.2

[6] V. A. J. M. Sleiffer et al., Proc OFC'13, OW1I.5

[7] P. J. Roberts, Optics Express, 13, 236-244 (2005)

[8] M. N. Petrovich et al., Proc ECOC'12, Th.3.A.5.

[9] J. Wooler et al., Proc $O F C^{\prime} 13$, OM3I.5 